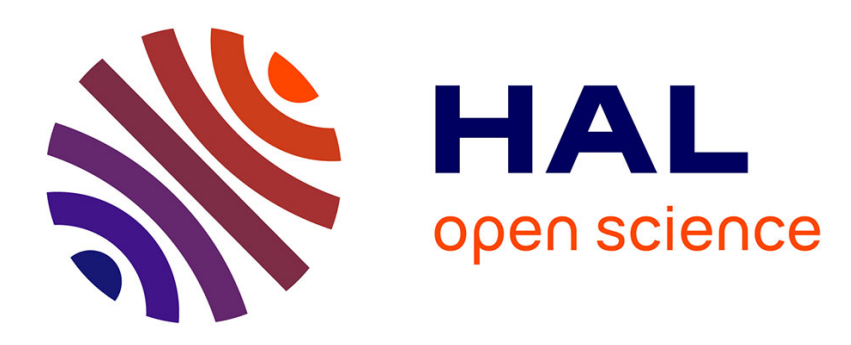

\title{
Age distribution of trees in stationary forest system
}

Petri P. Kärenlampi

\section{To cite this version:}

Petri P. Kärenlampi. Age distribution of trees in stationary forest system. Journal of Theoretical Biology, 2011, 270 (1), pp.13. 10.1016/j.jtbi.2010.11.019 . hal-00656341

\section{HAL Id: hal-00656341 https://hal.science/hal-00656341}

Submitted on 4 Jan 2012

HAL is a multi-disciplinary open access archive for the deposit and dissemination of scientific research documents, whether they are published or not. The documents may come from teaching and research institutions in France or abroad, or from public or private research centers.
L'archive ouverte pluridisciplinaire HAL, est destinée au dépôt et à la diffusion de documents scientifiques de niveau recherche, publiés ou non, émanant des établissements d'enseignement et de recherche français ou étrangers, des laboratoires publics ou privés. 


\section{Author's Accepted Manuscript}

Age distribution of trees in stationary forest system

Petri P. Kärenlampi

PII:

S0022-5193(10)00604-1

DOI: doi:10.1016/j.jtbi.2010.11.019

Reference: YJTBI 6241

To appear in:

Journal of Theoretical Biology

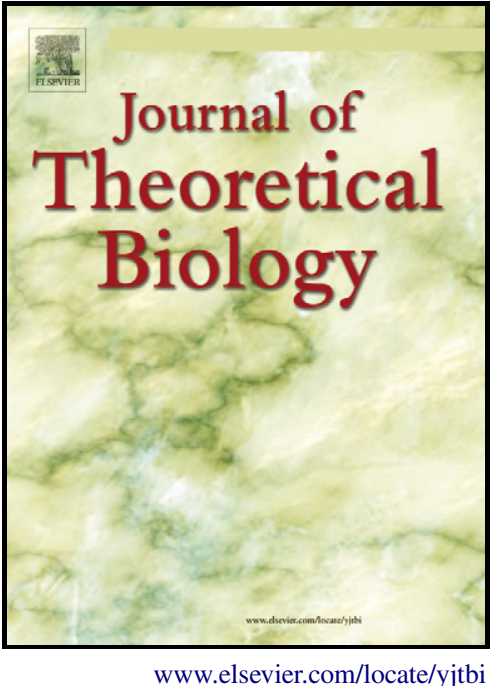

Received date: $\quad 30$ June 2010

Revised date: 28 September 2010

Accepted date: $\quad 10$ November 2010

Cite this article as: Petri P. Kärenlampi, Age distribution of trees in stationary forest system, Journal of Theoretical Biology, doi:10.1016/j.jtbi.2010.11.019

This is a PDF file of an unedited manuscript that has been accepted for publication. As a service to our customers we are providing this early version of the manuscript. The manuscript will undergo copyediting, typesetting, and review of the resulting galley proof before it is published in its final citable form. Please note that during the production process errors may be discovered which could affect the content, and all legal disclaimers that apply to the journal pertain. 


\title{
Age Distribution of Trees in Stationary Forest System
}

\author{
Petri P. Kärenlampi \\ University of Eastern Finland \\ Box 111 \\ FIN-80101 Joensuu \\ petri.karenlampi@joensuu.fi \\ tel. $+358-50-3711851$ \\ fax $+358-13-2514422$
}

\section{Abstract}

A statistical theory for the age distribution of spatially dominant trees in a stationary forest system is developed. The result depends whether or not mortality is spatially correlated, as well as whether or not the stand boundaries are predetermined. In the case of spatially non-correlated mortality, the tree age distribution is an exponential with survival rate as the base. In the case of spatially correlated mortality within a stand with pre-determined boundaries, the age distribution within the stand is an exponential with natural base. For a small stand, the median life span of the stand is inversely proportional to the number of trees $(n)$; the median life span in relation to stand closure time is inversely proportional to $n \ln (n)$. For a large stand, the stand life does not extend to the closure time.

The behavior of a forest system without fixed stand boundaries depends on the dimensionality of the system. In the case of a one-dimensional system, the longevity distribution is exponential, most of the trees however having the same longevity. Consequently, the probability density of tree age is constant. However, the probability mass of size of catastrophe destroying a particular tree is evenly distributed. This is due to trees being rapidly born on empty areas in the beginning of the life cycle, and clusters rapidly growing into larger ones close to the end of tree life.

Keywords:

tree mortality, stand boundaries, catastrophes, forest fires, self-organized criticality 
Petri P. Kärenlampi, November 12, 2010

\section{Introduction}

Late 1970's, Van Wagner [1] developed a simple model on the age distribution of trees within a forest. He started with the assumptions of random fire appearance, constant size of simultaneously burned area, and instantaneous regeneration within a stand. An exponential tree age density function was produced, the survival rate as base. Such a function behaves in a manner resembling the natural exponential of negative tree age, which Van Wagner found easier to use in computations.

According to computer simulations by Boychuck et al. [2], the age distribution of trees or stands within a forest area in general is not stationary. This certainly is the case: the probability density function of stand age within a forest system being constant, the actual distribution of stand age varies with time, unless the size of the system approaches infinity. A finite-size system, however, may well be stationary in the probabilistic sense.

The distribution of probability density of tree age is not necessarily stationary. It may evolve due to a variety of external factors, changes in climate and human activity being among the most influential $[3,4,5,6]$. The probability density of tree age also may evolve due to reasons internal to the forest system. Some periodic effects certainly take place, already due to weather seasons within any year. Obviously, the time scale of a year forms a lower cutoff for any stationarity of a forest system. 
Periodic events certainly take place within a stand with predetermined boundaries, consisting of trees with finite life span. The stand boundaries, however, may be subject to change, due to the internal dynamics of the forest system $[7,8,9,10]$. Even if the stand boundaries would be stationary, periodic changes within stands may compensate each other within a large system, such a system possibly retaining a large-scale stationary state, at least in the probabilistic sense.

Some observers report tree age distributions with a nonzero mode value [11]. Such observation cannot describe a forest system with stationary tree age distribution. The reason is that any sexually propagated tree starts its life at age zero. Then, the probability density of tree age may only decrease as a function of tree age, due to mortality. In other words

$$
\frac{d P(A)}{d A} \leq 0
$$

The simple model by Van Wagner [1] certainly satisfies this requirement. The data reported by Dai et al. [11] does not. Consequently, the latter kind of observations may apply to a stand with evolving age structure, not to a forest system with stationary age distribution.

We intend to discuss age distribution of trees within a forest which is in a largescale stationary state. We will first discuss a case where mortality is spatially uncorrelated. Then, we investigate the case where mortality is spatially correlated within a pre-determined stand, restrained by watersheds, mountain regions, or otherwise permanently treeless areas. Then, the age distribution of trees within a one-dimensional stand without pre-determined boundaries is computed. Finally, we discuss a one-dimensional forest system consisting of stands interacting in a probabilistic manner. 


\section{Spatially Uncorrelated Mortality}

Let us discuss a lattice model of a forest. Sites within the lattice may be either empty, or they may contain a tree. First we define two temporal probabilities. The probability that a tree appears on a previously empty site is $p$. The probability that a tree dies, and the site becomes empty, is $\mathrm{m}$. The density of trees within the lattice is $\rho$.

At the stationary state, the number of new trees must, on the average, equal the number of dying trees. Thus

$$
(1-\rho) p=\rho m
$$

At stationary state, the age distribution of trees must be essentially constant in time. The probability density of tree age is denoted as $p(A)$. Observations indicate that the mortality $m$ is essentially independent of age $[12$, cf. $13,14,15]$, in which case the probability density function of tree age becomes

$$
P(A)=-\ln (1-m)(1-m)^{A}
$$

Consequently, the age corresponding to fraction $q$ of trees is

$$
A_{q}=\frac{\ln (1-q)}{\ln (1-m)}
$$

Thus we find that the age corresponding to a particular fraction of trees is interdependent with mortality, and the mortality can be written as

$$
m=1-(1-q)^{\frac{1}{A_{q}}}
$$


Now we recognize that the density of trees within the forest, depending solely on the ratio $\frac{m}{p}$, can also be written in terms of the age

$$
\rho=\frac{1}{1+\frac{m}{p}}=\frac{1}{1+\frac{1-(1-q)^{\frac{1}{A_{q}}}}{p}}
$$

\section{Spatially Correlated Mortality}

All the quantities discussed so far have been taken as spatially uncorrelated. The mortality of trees may be spatially correlated. Catastrophes killing trees may spread from one site to the neighbouring sites. Among other causes, a spatially correlated catastrophe may be due to fire, earthquake, avalanche, insect damage, or human activity. Let us discuss an area where $n$ connected sites become simultaneously unoccupied. In other words, the local tree density within a stand suddenly drops to zero. Later on, the appearance rate of occupied sites within the stand is

$$
\frac{d o}{d t}=n p(1-\rho)
$$

from which the number of occupied sites can be solved

$$
o=n\left(1-e^{-p t}\right)
$$

The time derivative of Eq. (8) is

$$
\frac{d o}{d t}=n p e^{-p t}
$$


At time $t$, the age of any tree established at time $t_{o}$ is $\tau=t-t_{o}$. Change of variables in Eq. (9) results as

$$
\frac{d o}{d \tau}=-n p e^{-p(t-\tau)}
$$

and the density function of tree age within the stand becomes

$$
P_{s}(\tau)=\frac{p e^{p \tau}}{e^{p t}-1}=\frac{p e^{-p(t-\tau)}}{\rho(t)}
$$

According to Eq. (11), the age distribution of trees within a stand depends on the age of the stand, i.e. on the time elapsed since trees were last time catastrophically destroyed. The stand age places an upper cut-off to the distribution of tree ages, and in many cases there is a significant probability mass of tree age close to the stand age.

A related question is the lifetime of a stand. In the case of a forest susceptible to catastrophes, there must be some probability per time unit for the initiation of a catastrophe within an occupied site. Let us denote this probability $f$. Then, the probability of stand survival at age $t$ is

$$
S(t)=\prod_{u=0}^{t}[1-f n \rho(u)]=\prod_{u=0}^{t}\left[1-f n\left(1-e^{-p u}\right)\right]
$$

Up to the first order of $f n$ this survival probability can be approximated as

$$
S(t) \approx 1-f \sum_{u=o}^{t} o(u)=1-f n \sum_{u=o}^{t}\left(1-e^{-p u}\right) \approx 1-f n\left(t-\frac{1-e^{-p t}}{p}\right)=1-f n\left(t-\frac{\rho(t)}{p}\right)
$$

where the latter term corresponds to accumulated probability of catastrophe occurrence. An upper cutoff for the lifetime of the stand is defined where such accumulated stand mortality approaches unity. The result is 


$$
t_{\max }(n)=\frac{1}{f n}+\frac{1}{p}
$$

Within time range between zero and $t_{\max }$, the probability density of stand mortality $f n\left(1-e^{-p t}\right)$ is normalized as such. The probability density of stand age can be computed using Eq. (13) as

$$
P(\tau)=\int_{t_{\max }}^{\tau} S(t) d t=\left.\right|_{t_{\max }} ^{\tau}\left[\left(1+\frac{f n}{p}\right) t-\frac{f n}{2} t^{2}+\frac{f n}{p^{2}} e^{-p t}\right]
$$

which can be normalized in a standard but tedious way. Eq. (15) naturally satisfies requirement (1).

The median life span of a stand is

$$
t_{0.5}(n)=\frac{0.5}{f n}+\frac{\rho(t)}{p}
$$

Eq. (16) implies that unless the stand is very large, the life span is inversely proportional to the number of trees. In the case of a very large stand, the life span depends on how fast trees grow into the area.

Let us then discuss the relation of stand age and occupation. During first time step of a stand, the probability that the first tree appears is $\mathrm{np}$. With $\mathrm{t}$ consequent time steps, the probability accumulates to tnp. The median time required for the appearance of the first tree is

$$
T_{0.5}(1, n)=\frac{0.5}{n p}
$$

and the median time required for the appearance of the next tree when o sites are occupied is

$$
T_{0.5}(o+1, n)=\frac{0.5}{(n-o) p}
$$


Consequently, the median time for a stand of $\mathrm{n}$ sites to became occupied by trees

is

$$
T_{0.5}(n, n)=\frac{0.5}{p} \sum_{q=1}^{n} \frac{1}{q} \approx \frac{\ln (n)}{2 p}
$$

Now, the median life span of a stand, in relation to the time scale required for it to become occupied by trees is

$$
\frac{t_{0.5}(n)}{T_{0.5}(n, n)}=\frac{1}{\ln n}\left[\frac{p}{f} \frac{1}{n}+2 \rho\right]
$$

where the latter term must be rather small for a stand with a large number of trees. It will, however, dominate Eq. (20) in the case the catastrophe occurrence probability $f n$ is high in relation to $p$. Such large stands typically become destroyed before getting fully occupied. It is worth noting that the stationary age distribution within a forest system with predetermined stand boundaries depends on the size distribution of the stands.

\section{A One-Dimensional Stand without Pre-Determined Boundaries}

The boundaries of a stand are not necessarily restricted by mountains or watersheds. Trees growing on the boundaries increase the size of the particular cluster. Growing trees also may fill a gap between two stands unifying the two into one larger stand. A catastrophe occurring on a neighbouring stand may temporarily exclude such a possibility. Along with a catastrophe occurring within a stand, its existence may cease.

A stand, or a cluster of trees, in general may have a variety of geometric configurations. However, in one dimension, the only possible geometry is that of a 
string. This makes it possible to produce an approximation for the age distribution within a one-dimensional forest.

Let us discuss the age distribution within a stand (or cluster) of size $\mathrm{n}$. The median time required for the appearance of the m:th tree is produced using Eq. (18) as

$$
T_{0.5}(m, n)=\frac{0.5}{p} \sum_{o=0}^{m-1} \frac{1}{n-o}=\frac{0.5}{p} \sum_{q=n-m+1}^{n} \frac{1}{q}
$$

It is worth noting that Eq. (21) can be seen as an upper approximation. The boundaries of the stand not being predetermined, there are more opportunities for the placement of the first trees. However, these additional opportunities cease along with the growth of trees, and Eq. (21) can be considered a rather accurate approximation for large clusters.

Eq. (21) gives an approximation for the median time a string of $n$ sites becomes full. The stand boundaries not being pre-determined, the stand will grow larger with additional time. Thus, the median age of the $m$ :th tree on a stand of $n$ trees is

$$
\begin{aligned}
& \tau_{0.5}(m, n)=T_{0.5}(n, n)-T_{0.5}(m, n)= \\
& \frac{1}{2 p}\left[\sum_{q=1}^{n} \frac{1}{q}-\sum_{q=n-m+1}^{n} \frac{1}{q}\right]=\frac{1}{2 p} \sum_{q=1}^{n-m} \frac{1}{q} \approx \frac{\ln (n-m)}{2 p}=\frac{\ln (n)+\ln (1-m / n)}{2 p}
\end{aligned}
$$

Now, the probability density of tree age within the stand can be given as

$$
P_{s}(\tau)=-p(m) \frac{d m}{d \tau}=\frac{1}{n} 2 p(n-m)=\frac{2 p e^{2 p \tau}}{n}
$$

The negative sign in Eq. (23) corresponds to the positive direction of tree age being the reverse of the positive direction of the tree number $m$. 
The median age of trees within the stand is readily calculable from Eq. (22). It is

$$
\tau\left(\frac{n}{2}, n\right)=\frac{\ln (n)-\ln (2)}{2 p} \approx \frac{\ln (n)}{2 p}
$$

In other words, the median age of trees significantly differs from the stand age only in the case of rather small stands (cf. Eq. (19)). The same applies to the mean age of trees within the stand (Eq. (25)).

$$
\bar{\tau} \approx \frac{\ln (n)-1+1 / n}{2 p} \approx \frac{\ln (n)}{2 p}
$$

\section{Several Stands Interacting Probabilistically}

The internal dynamics of a stationary forest system depends on its dimensionality. It probably can be determined analytically only in the case of a one-dimensional system.

The cluster size distribution within a one-dimensional forest system can be derived on the basis of conditions for the stationary state $[9,16]$. The local appearance probability of a cluster of size $n$, normalised by the size of the entire system, is

$N(n)=\frac{1-\rho}{(n-1)(n-2)} \approx(1-\rho) n^{-2}$

The corresponding unnormalized probability of a tree to belong to a cluster of size $\mathrm{n}$ is

$n N(n)=\frac{n(1-\rho)}{(n-1)(n-2)} \approx(1-\rho) n^{-1}$ 
In case the cluster size distribution would happen to include an infinite cluster, Eq. (27) could not be normalized. However, there is an upper cutoff for the cluster size. This is due to the fact that the probability of a catastrophe occurring within a stand during its time of growth, which is given in Eq. (19), is $f \sum_{0}^{T_{05}(n, n)} m(T)=f \sum_{0}^{T_{05}(n, n)} m(T)=f \sum_{t=0}^{T_{0.5}(n, n)} n\left(1-e^{-p t}\right) \approx \frac{f}{2 p} n \ln (n)$

This probability approaching 1 , we find the upper cluster size cutoff as

$$
n_{\max } \ln \left(n_{\max }\right) \approx 2 \frac{p}{f}
$$

Considering this cutoff, Eq. (27) can be normalised. The probability density of a tree to belong to a cluster of size $n$ is $P(n) \approx \frac{1}{n \ln \left(n_{\text {max }}\right)}$

The mean and median values of tree age within a stand (Eqs. (24) and (25)) closely resembling the stand age (Eq. (19)), the stand age can be taken to characterize tree age within any stand. Consequently, the probability density of tree age within the entire forest system is

$$
P(\tau)=P(n) \frac{d n}{d \tau} \approx \frac{2 p}{\ln \left(n_{\max }\right)}
$$

Surprisingly, we find that the probability density of tree age is constant - it does not depend on tree age! For a randomly selected individual tree, any age between unity and $\frac{\ln \left(n_{\max }\right)}{2 p}$ is equally probable! 
At first glance, the result appears somewhat strange. It indicates that nearly all trees survive up to maximum age. The natural explanation is that trees grow rapidly in empty space. On the other hand, clusters grow in rate proportional to cluster size, or $\frac{d n}{d \tau} \approx 2 p n$. Consequently, large clusters grow fast towards the maximum size, and the large clusters are most vulnerable to catastrophes.

An apparent contradiction to the result is that size distribution of catastrophes (or "fires") is given by $n N(n)$. The probability of a particular tree to become involved in a catastrophe of size $n$ is $n^{2} N(n)$, which is constant. In other words, the probability density of a tree to become involved in a fire of size $n$ is

$$
F(n)=\frac{1}{n_{\max }}
$$

The probability density of a tree to become destroyed at age $t$ is

$$
F(\tau)=F(n) \frac{d n}{d \tau}=\frac{2 p n}{n_{\max }}=\frac{2 p e^{2 p \tau}}{n_{\max }}
$$

We find from Eq. (33) that the probability density of terminal tree age within the entire one-dimensional forest system is the same as the age distribution of trees within a cluster (Eq. (23)), except that the denominator now contains $n_{\max }$ instead of n. Consequently, practically all trees live up to age $\ln \left(n_{\max }\right) / 2 p$.

Interestingly, the above results apply regardless the density of trees within the forest. The tree density can be produced by integrating Eq. (27), resulting as 
$\frac{\rho}{(1-\rho)} \approx \ln \left(n_{\max }\right)$

Thus we find that the upper cutoff cluster size distribution, as well as the tree age distribution, is related to tree density. The upper cutoff cluster size in turn is related to the parameter ratio $\frac{f}{p}$ as indicated in Eq. (29).

\section{Summary of Results}

Our treatment regarding spatially uncorrelated mortality of trees appears to be related to the simple model by Van Wagner and followers [1, 14, 4]. He started with the assumptions of random fire appearance and instantaneous regeneration within a stand. From the age perspective, the latter assumption makes a Van Wagner's stand analogous to a tree in our treatment. Correspondingly, an exponential age distribution was produced, the survival rate as base - a finding which agrees with our Eq. (3). A truncated Taylor series of a natural exponential of the negative of mortality yields the survival rate, which justified Van Wagner to use the natural exponential in computations. Such a function is non-equivalent to our result regarding spatially correlated mortality, with tree appearance rate in the exponent (Eq. (11)). Further, Eq. (11) is an exponential of positive tree age, whereas the approximation by Van Wagner is an exponential of the negative of tree age $[1,14$, 4].

Within our treatment of spatially correlated mortality, the density function of tree age within a stand depends on stand age, according to Eq. (11). The life span of a stand then depends on the size of the stand according to Eq. (16). In other words, there is a stand-size effect on the density function of tree age. Interestingly, no size 
effect on tree age distribution appears in the study of Van Wagner [1], due to negligence of spatial mortality correlations.

The situations discussed above may be regarded as classical forestry problems, mortality being either spatially correlated or uncorrelated, and new trees being established either instantly or with a finite rate, within fixed stand boundaries. It is worth noting that real forest systems typically include both spatially correlated and uncorrelated mortality.

The dynamics of the system changes considerably when stand boundaries become determined by the appearance and disappearance of trees. The stand size distribution becomes a power law, as does the size distribution of catastrophes. In general, the critical exponents tend to depend on system dimensionality $[8,17]$. Presumably the age distribution of trees within such a forest system also depends on dimensionality.

We have above investigated the one-dimensional case in detail. The density of tree age within a stand without pre-determined boundaries is an exponential function of tree age with positive exponent (Eq. (23)). This, apart from the case of rather small stands, accumulates the probability mass into the vicinity of the stand age. Thus the first moment of the tree age distribution within a stand is close to the stand age. Still more interestingly, the tree age density within the entire forest system is approximately constant (Eq. (31)). In other words, almost all trees live to the upper cutoff tree age.

It is of interest how the results regarding the self-organized critical forest system would change in case of a catastrophe being able to jump over some size of a vacation. In other words, a fire may able to destroy a porous stand - even a one- 
dimensional porous stand. This changes the computation of the upper cutoff of cluster size. Instead of cluster filling time as given in Eq. (19), the cluster becomes susceptible to fire at a critical tree coverage $\left(\frac{m}{n}\right)_{c}$, and the upper limit of time summation in Eq. (28) has to be changed to one corresponding to Eq. (21). Consequently, the logarithm of critical porosity enters the Equation defining upper cutoff size of a porous cluster as $-n_{\max } \ln \left(1-\left(\frac{m}{n}\right)_{c}\right) \approx 2 \frac{p}{f}$

The one-dimensional case allowing an analytical treatment, the two-dimensional case probably does not. This is due to widely varying cluster geometries. A few numerical investigations have been conducted. The results of Sinha-Ray et al. [18], indicate that the probability density of tree age is an exponential function of the negative of tree age.

\section{Discussion}

The discussion of forest stands with pre-determined boundaries obviously relates to physical reality in cases where such boundaries exist. Stand boundaries may become determined by human activity. Even in the absence of human determination, geophysical heterogeneity appears in the form of mountain ranges, ridges, watersheds, or even temporal barriers like rainy seasons. In the case where stand boundaries are not predetermined, contingency rules.

The one-dimensional forest with stands interacting probabilistically displays a power-law distribution of cluster sizes, as well as in the size distribution of catastrophes (Eqs. $(26,27))$. Consequently, it displays self-similarity with respect 
to change in magnification of observation. This size distribution however has an upper cutoff at $n_{\max }$. The mean and median of the distribution do not diverge, and thus the cluster size distribution, as well as the distribution of the size of the catastrophes, does have a characteristic scale.

Any finite physical system with self-similarity has both a lower and an upper cutoff. A self-organized critical system has criticality as an attractor: seeks itself to the state where avalanches distribute according to a power law, despite change in parameter values $[19,20]$. Changing the parameter ratio $\frac{f}{p}$ results in changes in the characteristics of the one-dimensional forest system: the upper cutoff cluster size changes according to Eq. (29), and the tree density changes according to Eq. (34). The time scale of tree life changes as said in Eq. (31). However, the size distribution of catastrophes becomes a power-law (Eq. (27)) without tuning the values of parameters, including the initial tree density. The system is robustly attracted to such behaviour. It is self-organized critical.

Some amount of confusion appears in the literature. Drossel and Schwabl [8, 9, 10] claim that a forest fire model becomes critical at the limit $\frac{f}{p} \rightarrow 0$. This may be related to an assumption of instantaneous burning, used in this paper, but not in the papers of Drossel and Schwabl. The requirement of the zero limit arises from the "double separation of time scales". On the one hand, catastrophes must happen faster than trees grow. On the other hand, trees must have time to grow and form clusters before a catastrophe occurs. In our treatment, the first condition, often denoted as "slow driving", is taken care of by instantaneous spreading of any catastrophe. The latter condition enters our discussion through the assumption of 
relatively large clusters. The system is here concluded to be self-organized critical apart from the limit $\frac{f}{p} \rightarrow 0$.

It is, however, obvious that different authors disagree about the meaning of criticality. Instead of a system which, for a range of initial conditions and parameter values, becomes attracted to a state with self-similar size distribution of avalanches as proposed by Bak [19, 20], Drossel and Schwabl $[8,9,21]$ denote a state without upper cutoff in the size distribution of avalanches as a "critical point". Simultaneously, Drossel and Schwabl admit that a system of finite size cannot reach such a critical point. In later papers, Drossel admits that a power-law distributed avalanche size as an attractor does indicate self-organized criticality $[22,17,21]$. It also appears that SOC may arise not only in a random process, but also in a deterministic process, in the presence of separation of energy scales [7, 21]. Such a critical state however is found not to be chaotic [7].

It actually has been claimed that no system without a conservation law can be truly self-organized critical [23]. In other words, dissipating systems do not appear to become stationary even at the thermodynamic limit, but hover around a critical point. Such systems are proposed to be denoted as "self-organized quasi-critical" [23]. On the other hand, Fock-space formalism does result as a critical forest fire system [24].

Another issue of interest is, to which degree the outcomes of the simple models of self-organized critical systems correspond to real behaviour of forest ecosystems. In the case of forest fires, observations indicate that the size distribution of fires indeed appears as some kind of a power law [25, 26, 27, 28]. However, a single exponent does not necessarily apply to the entire scaling range. The entire range can be covered in terms of a composite power law with different exponents for 
small and large fires $[26,27,29,30,28]$. There are at least two possible explanations for this kind of behaviour.

Firstly, qualitatively different fires may exist due to finite-size effects [31, 25]. In other words, stand boundaries may be partly predetermined. The finite-size effects may be due to geophysical heterogeneity like mountain ranges, ridges, watersheds, or temporal barriers like rainy seasons [5, 27, 29]. Human activity also may induce stand boundaries which are predetermined from the viewpoint of wildfires. Finite-size effects induce periodicity in the occurrence of catastrophes [3, $5,6,32]$, whereas avalanches in true self-organized critical systems do not occur periodically $[19,20,23]$. Finite size of a stand appears to be analogous to tree appearance function without an exponential tail [33]. Finite-size effects may be anomalous in the case of quasi-critical systems [34, 35, 23].

The second possible explanation is that fires as such may differ qualitatively. Fires may be surface fires, ground fires, or mixtures of these [28]. A phenomenological model, based on such classification, has succeeded in producing a composite power law with different exponents for small fires and large fires [28].

Both of the explanations for observed fire size distribution differing from a simple exponential may be correct, even simultaneously. In the mind of the present author, the theory of finite-size effects [31] appears more tempting, since it has been established without phenomenology. Within this view, some of the stand boundaries being predetermined, the tree age distribution may be something between distributions due to pre-determined stand boundaries, and those appearing as a consequence of self-organized critical dynamics. The latter depend on dimensionality. The transition between these two kinds of behaviours might be an interesting topic of further study. 
Petri P. Kärenlampi, November 12, 2010

\section{References}

[1] C.E. Van Wagner, Age-class distribution and the forest fire cycle, Can. J. For. Res. 8 (1978) 220-227.

[2] D. Boychuk, A.H. Perera, M.T. Ter-Mikaelian, D.L. Martell, C. Li, Modelling the effect of spatial scale and correlated fire disturbances on forest age distribution, Ecological Modelling 95 (1997) 145-164.

[3] E.A. Johnson, Fire recurrence in the subarctic and its implications for vegetation composition, Canadian Journal of Botany 57 (1978) 1374-1379.

[4] E.A. Johnson, C.E. Van Wagner, The theory and use of two fire history models, Can. J. For. Res. 15 (1985) 214-220.

[5] J.S. Clark, Fire and climate change during the last 750 years in Northwestern Minnesota, Ecol Monogr. 60 (1990) 135-159.

[6] J.S. Clark, Ecological disturbance as a renewal process: theory and application to fire history, Oikos 56 (1989) 17-30.

[7] K. Chen, P. Bak, M.H. Jensen, A deterministic critical forest fire model, Phys. Lett. A 149 (1990) 207-210.

[8] B. Drossel, F. Schwabl, Self-organized critical forest fire model, Phys. Rev. Lett. 69 (1992) 1629-1632.

[9] B. Drossel, S. Clar, F. Schwabl, Exact results for the one-dimensional selforganized critical forest-fire model, Phys. Rev. Lett. 71 (1993) 3739-2742.

[10] B. Drossel, F. Schwabl, Formation of space-time structure in a forest-fire model, Physica A 204 (1994) 212-229.

[11] D. Limin, S. Weizhong, D. Hongbing, D. Hongcai, Z. Yiping, J. Ping, Age structure of Tilia broad-leaved Korean pine forest on northern slope of Changbai Mountain, Journal of Forestry Research 9 (1998) 182-185.

[12] D. Laarmann, H. Korjus, A. Sims, J.A. Stanturf, A. Kiviste, K. Köster, Analysis of forest naturalness and tree mortality patterns in Estonia, Forest Ecology and Management 258 (2009) S187-S195.

[13] C.E. Van Wagner, Conditions for the start and spread of crown fire, Can. J.

For. Res. 7 (1977) 23-34.

[14] J. Yarie, Forest fire cycles and life tables: a case study from interior Alaska, Canadian Journal of Forest Research 11 (1981) 554-562

[15] K. Umeki, Tree mortality of five major species on Hokkaido Island, Northern Japan, Ecological Research 17 (2002) 575-589.

[16] P. Bak, M. Paczuski, Theory of the one-dimensional forest-fire model, Phys. Rev. E 48 (1993) R3214-R3216.

[17] S. Clar, B. Drossel, F. Schwabl, Scaling laws and simulation results for the self-organized critical forest-fire model, Phys. Rev. E 50 (1994) 1009-1018.

[18] P. Sinha-Ray, L.B.de Água, H. Jeldtoft Jensen, Threshold dynamics, multifractality and universal fluctuations in the SOC forest-fire: facets of an autoignition model, Physica D 157 (2001) 186-196.

[19] P. Bak, M. Paczuski, Complexity, contingency and criticality, Proc Natl Acad Sci U S A 92 (1995) 6689-6696.

[20] P. Bak, How Nature Works. The Science of Self-Organized Criticality, Copernicus, NY, 1996.

[21] B. Drossel, Self-organized criticality due to a separation of energy scales. Physica A 236 (1997) 309-320.

[22] B. Drossel, S. Clar, F. Schwabl, Universality in the one-dimensional selforganized critical forest-fire model, Z. Naturforsch. 49 (1994) 856-860.

[23] J.A. Bonachela, M.A. Muñoz, Self-organization without conservation: true or just apparent scale-invariance? J. Stat. Mech. (2009) P09009.

[24] H. Patzlaff, S. Trimper, Analytical approach to the forest-fire model, Phys. Lett. A 189 (1994) 187-192.

[25] B.D. Malamud, G. Morein, D.L. Turcotte, Forest fires: an example of selforganized critical behaviour, Science 281, (1998) 1840-1842. 
[26] C. Ricotta, G. Avena, M. Marchetti, The flaming sandpile: self-organized criticality and wildfires, Ecological Modelling 119 (1999) 73-77.

[27] C. Ricotta, M. Arianoutsou, R. Di'az-Delgado, B. Duguy, F. Lloret, E. Maroudi, S. Mazzoleni, J.M. Moreno, S. Rambal, R. Vallejo, A. Va'zquez, Self-organized criticality of wildfires ecologically revisited, Ecological Modelling 141 (2001) 307311.

[28] J. Lin, S. Rinaldi, A derivation of the statistical characteristics of forest fires, Ecological Modelling 220 (2009) 898-903.

[29] W.J. Reed, K.S. McKelvey, Power-law behaviour and parametric models for the size-distribution of forest fires, Ecological Modelling 150 (2002) 239-254.

[30] C. Ricotta, Fractal size distributions of wildfires in hierarchical landscapes: Natura facit saltus? Comments, Theoretical Biology 8 (2003) 93-101.

[31] K. Schenk, B. Drossel, S. Clar, F. Schwabl, Finite-size effects in the selforganized critical forest-fire model, Eur. Phys. J. B 15 (2000) 177-185.

[32] J.E.S. Socolar, G. Grinstein, C. Jayaprakash, On self-organized criticality in nonconserving Systems, Phys. Rev. E 47 (1993) 2366-2376.

[33] B. Drossel, Self-organized criticality and synchronization in a forest-fire model. Phys. Rev. Lett. 76 (1996) 936-939.

[34] G. Pruessner, H.J. Jensen, Broken scaling in the forest-fire model, Phys. Rev. E 65 (2002) 056707.

[35] P. Grassberger, Critical behaviour of the Drossel-Schwabl forest fire model, New J. Phys. 4 (2002) 17.1-17.15 